\title{
Ciliary proteins Bbs8 and Ift20 promote planar cell polarity in the cochlea
}

\author{
Helen L. May-Simera ${ }^{1, *, \neq}$, Ronald S. Petralia ${ }^{2}$, Mireille Montcouquiol ${ }^{3}$, Ya-Xian Wang ${ }^{2}$, Katherine B. Szarama ${ }^{1,4}$, \\ Yun Liu ${ }^{5}$, Weichun Lin ${ }^{5}$, Michael R. Deans ${ }^{6}$, Gregory J. Pazour ${ }^{7}$ and Matthew W. Kelley ${ }^{1}$
}

\begin{abstract}
Primary cilia have been implicated in the generation of planar cell polarity (PCP). However, variations in the severity of polarity defects in different cilia mutants, coupled with recent demonstrations of noncilia-related actions of some cilia genes, make it difficult to determine the basis of these polarity defects. To address this issue, we evaluated PCP defects in cochlea from a selection of mice with mutations in cilia-related genes. Results indicated notable PCP defects, including mis-oriented hair cell stereociliary bundles, in Bbs8 and Ift20 single mutants that are more severe than in other cilia gene knockouts. In addition, deletion of either Bbs 8 or Ift2O results in disruptions in asymmetric accumulation of the core PCP molecule Vangl2 in cochlear cells, suggesting a role for Bbs8 and/or Ift20, possibly upstream of core PCP asymmetry. Consistent with this, coimmunoprecipitation experiments indicate direct interactions of Bbs8 and Ift20 with Vangl2. We observed localization of $\mathrm{Bbs}$ and Ift proteins to filamentous actin as well as microtubules. This could implicate these molecules in selective trafficking of membrane proteins upstream of cytoskeletal reorganization, and identifies new roles for cilia-related proteins in cochlear PCP.
\end{abstract}

KEY WORDS: Actin, Cilia, Cochlea, Microtubules, Polarity, Mouse

\section{INTRODUCTION}

Primary cilia were originally thought to be vestigial organelles without specific function. However, recent research has demonstrated that defects in primary cilia cause a range of developmental defects and human disorders collectively termed 'ciliopathies' (Lee and Gleeson, 2011; Waters and Beales, 2011), with Bardet-Biedl syndrome (BBS) considered to be an archetype for these disorders (Forsythe and Beales, 2013). Cilia are microtubule-based appendages continuous with the cell membrane but extending away from the cell surface (Fisch and Dupuis-Williams, 2011). At the core of each cilium is the ciliary axoneme, a microtubule-based structure containing

\footnotetext{
Section on Developmental Neuroscience, Laboratory of Cochlear Development, National Institute on Deafness and other Communication Disorders, National Institutes of Health, Bethesda, MD 20892, USA. ${ }^{2}$ Advanced Imaging Core, National Institute on Deafness and other Communication Disorders, National Institutes of Health, Bethesda, MD 20892, USA. ${ }^{3}$ Planar Polarity and Plasticity Group, Institut National de la Santé et de la Recherche Médicale U862, Neurocenter Magendie, 33077 Bordeaux, France. ${ }^{4}$ Department of Cell and Molecular Biology, St. Jude Children's Research Hospital, Memphis, TN 38105, USA. 'Department of Neuroscience, UT Southwestern Medical Center, Dallas, TX 75235, USA ${ }^{6}$ Division of Otolaryngology-Head and Neck Surgery and Department of Neurobiology \& Anatomy, University of Utah School of Medicine, Salt Lake City, UT 84132, USA. 'Program in Molecular Medicine, University of Massachusetts Medical School, Worcester, MA 01605, USA.

*Present address: Neurobiology Neurodegeneration and Repair Laboratory, Retinal Cell Biology Degeneration Section, National Eye Institute, 6 Center Drive, Bethesda, MD 20892, USA.

‡Author for correspondence (maysimerah@nei.nih.gov)
}

Received 1 June 2014; Accepted 3 December 2014 either $9+2$ or $9+0$ microtubule doublets surrounded by soluble matrix and ciliary membrane. The proximal ends of these doublets are anchored to the basal body, a structure derived from the mother centriole following mitosis (Kobayashi and Dynlacht, 2011). The ciliary basal body is also a microtubule-organizing center that regulates ciliary and vesicular trafficking at the luminal surface (MaySimera and Kelley, 2012b; Moser et al., 2010). Finally, a transition zone located at the base of the ciliary axoneme and overlapping with the basal body plays a key regulatory role. All these components are considered to be part of the cilium, with disruption leading to ciliary defects. Many ciliary proteins have been identified based upon tight association with cilia; however, emerging evidence suggests that these proteins have additional, non-cilia-related functions. For example, intraflagellar transport (IFT) proteins have been reported at non-ciliary locations, including the membranous Golgi and dendrites of retinal neurons (Finetti et al., 2009; Sedmak and Wolfrum, 2010; Yuan and Sun, 2013). Similarly, Bbs proteins are implicated in actin cytoskeleton regulation (Hernandez-Hernandez et al., 2013; Tobin et al., 2008) and are localized at actin-rich structures in cultured cells and mouse cochleae (HernandezHernandez et al., 2013; May-Simera et al., 2009).

Cilia also contribute to intercellular signaling pathways, including the planar cell polarity (PCP) branch of the Wnt signaling pathway, but the current understanding of how cilia are associated with PCP signaling is unclear (Wallingford and Mitchell, 2011). Although intricately linked, the phenomenon of PCP is not completely synonymous with PCP signaling. PCP refers to the uniform orientation of cells within an epithelium (Simons and Mlodzik, 2008; Vladar et al., 2009). PCP signaling is the information flow that is required to achieve this orientation; it is also more narrowly defined as the system of signaling that produces asymmetric subcellular localization of core PCP proteins. Cilia were first implicated in PCP signaling after PCP-like phenotypes were identified in Bbs mutants (Ross et al., 2005). One theory is that cilia regulate a switch between $\mathrm{PCP}$ and canonical Wnt signaling via sequestration of signaling molecules near the basal body (Lienkamp et al., 2012; Simons et al., 2005; Veland et al., 2013). However, the causal relationship between the cilium and PCP signaling has not been elucidated (Wallingford and Mitchell, 2011). One complication is that the morphological response to PCP signaling is usually the localization of the primary cilium; therefore, mutations in ciliary proteins that affect ciliary location will affect PCP but not necessarily PCP signaling.

In vertebrates, a striking example of PCP is the uniform orientation of stereociliary bundles on mechanosensory hair cells of the inner ear (Ezan and Montcouquiol, 2013). Stereociliary bundles are composed of a specialized cilium, called the kinocilium, positioned adjacent to elongated actin-rich microvilli called stereocilia, based on historical convention. The stereociliary bundles are polarized and the appropriate positioning of kinocilia 

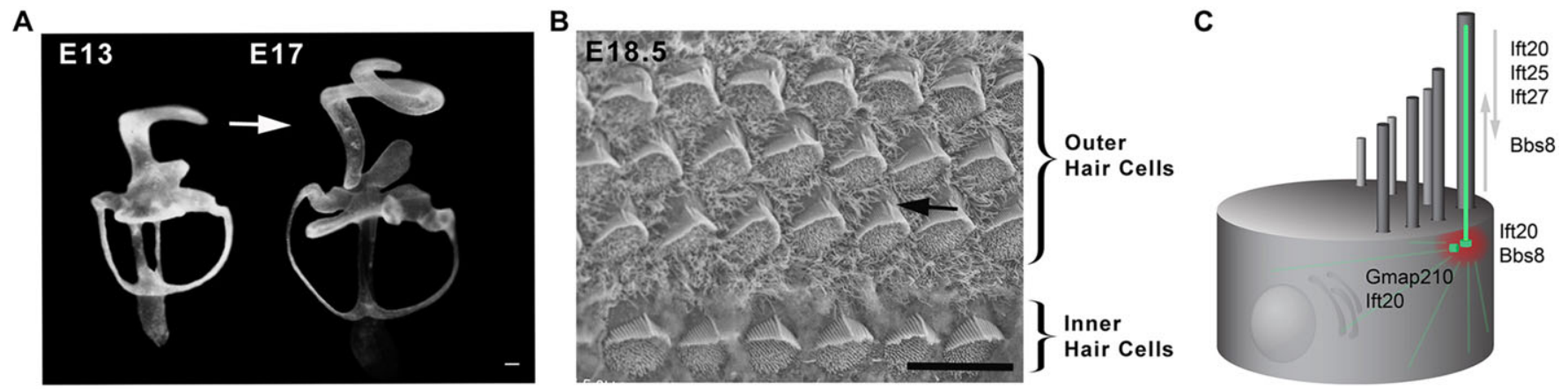

Fig. 1. Cochlea phenotypes in cilia mutants. (A) Lateral view of paint-filled inner ears showing extension of the cochlear duct (white arrow) E13-E17 [adapted from Morsli et al. (1998)]. (B) SEM of organ of Corti from E17 cochlea. Uniform alignment of stereociliary bundles on IHCs and on three rows of OHCs is evident by E17. The kinocilium is consistently localized at the vertex of each stereociliary bundle (black arrow). (C) Schematic representation of an individual hair cell depicting known localizations of cilia-related proteins for which mutants were analyzed. Microtubules are green; the basal body is red. Scale bars: 100 um in $\mathrm{A} ; 10 \mu \mathrm{m}$ in $\mathrm{B}$

relative to stereocilia is required for normal hair cell function. Although the precise cellular processes that mediate bundle orientation are still being elucidated, a key step is thought to be the directed migration of the kinocilium to a lateral position on the apical hair cell surface (Cotanche and Corwin, 1991; DenmanJohnson and Forge, 1999). Six 'core' PCP proteins are essential regulators of bundle orientation that, when mutated, lead to varying degrees of mis-orientation owing to disrupted intercellular signaling (Montcouquiol et al., 2003; Wang et al., 2006). Cilia-related proteins, including Ift 88 and Kif3a, have also been shown to be required for appropriate bundle orientation (Jones et al., 2008; Sipe and Lu, 2011). Interestingly, many of these mutants also demonstrate defects in outgrowth of the cochlear duct, a process that is believed to require convergent extension, a conserved morphogenetic process that is also regulated by the PCP pathway.

These studies implicate cilia and cilia-related genes in two PCPdependent processes: stereociliary bundle orientation and cochlear outgrowth. However, whether the phenotypes observed are a result of disruptions in the PCP process, the formation of cilia or both is unclear. To address these issues, we determined changes in stereociliary bundle PCP and cochlear outgrowth in mice with mutations in several, different classes of cilia-related genes. Our results suggest that a subset of cilia-related genes is required for trafficking of PCP molecules to the cell membrane in a ciliaindependent manner and, as such, might play a more global role in protein trafficking within the cell.

\section{RESULTS}

\section{Analysis of cilia-related mutants}

Cochlear outgrowth (Fig. 1A) and stereociliary bundle orientation (Fig. 1B), two aspects of inner ear development known to be mediated through the PCP pathway, were analyzed using mouse lines with mutations in cilia-related genes. We obtained early postnatal or late embryonic cochlear tissue from Ift20, Ift25 (Hspb11 - Mouse Genome Informatics), Ift27, Gmap210 (Trip11 ${ }^{\text {Gt(AJ0490)Wtsi }}$ - Mouse Genome Informatics) and $B b s 8$ (Ttc 8 - Mouse Genome Informatics) mutant mice. Ift20, Ift25 and Ift27 are IFT complex B proteins required for both anterograde and retrograde IFT (Fig. 1C) (Follit et al., 2009; Lucker et al., 2005). Ift20 has additional roles related to Golgi-based sorting and vesicle trafficking of ciliary cargo (Follit et al., 2006), whereas Gmap210 anchors Ift20 to the Golgi complex (Follit et al., 2008). Bbs8 is thought to function as an adaptor protein for cargo undergoing IFT (Blacque et al., 2004; Tadenev et al., 2011). Despite a high degree of functional conservation between these molecules in other contexts, phenotypic variation in cochlear extension and bundle morphology was observed (Table 1). Cochleae from Ift $27^{-1-}$ mutants were significantly shorter than in controls but displayed only mild bundle disruption. By contrast, Ift $25^{-/-}$and GMAP $210^{-/-}$cochleae were comparable to control littermates (supplementary material Fig. S1). Bbs $8^{-/-}$and Ift2 $0^{\text {cko/cko }}$ mutants displayed more extreme PCP phenotypes and are described below.

\section{Disruption of stereociliary polarity in $\mathbf{B b s 8}^{-1-}$ cochleae}

Analysis of cochleae from P0 $B b s 8^{-/-}$mice revealed stereociliary bundle-orientation defects and flattened or misshapen bundles (Fig. 2A,B), but cochlear length was unchanged (supplementary material Fig. S2A). Consistent with other PCP mutants, stereociliary bundles were rotated and kinocilia were misplaced or occasionally absent. Kinocilia were often separated from stereociliary bundles, suggesting a loss of coupling between the structures. To confirm these changes, samples were examined by scanning electron microscopy (SEM) (Fig. 2C-I). At higher magnification, detached kinocilia and flattened bundle morphologies were visible (compare Fig. 2E with Fig. 2F,G). To quantify overall changes in kinocilia position and bundle orientation, both features were charted in wildtype (WT) and $B b s 8^{-/-}$cochleae (Fig. 2J,K). Both were mildly disrupted in $B b s 8^{-1-}$ inner hair cells (IHCs), with most kinocilia and bundles still restricted to the lateral quadrant of the lumenal surface of hair cells. A more severe disruption was seen in outer hair cells (OHCs), where kinocilia and bundles were observed throughout the lumenal surface (Fig. 2J,K). Previous analyses of cochlear phenotypes in PCP mutants demonstrated variations in severity of bundle defects between each of the three rows of OHCs (Montcouquiol et al., 2003). However, a similar analysis in $B b s 8^{-/-}$ cochleae indicated similar levels of defects in each row of OHCs. The flattened bundle morphology was further characterized by measuring the area between the vertex and ends of the two arms of

Table 1. Cochlea phenotype of cilia mutants

\begin{tabular}{lllll}
\hline Protein & $\begin{array}{l}\text { Mouse } \\
\text { mutant }\end{array}$ & $\begin{array}{l}\text { Cochlea } \\
\text { extension }\end{array}$ & $\begin{array}{l}\text { Rotated } \\
\text { SCB }\end{array}$ & $\begin{array}{l}\text { Disrupted } \\
\text { SCB }\end{array}$ \\
\hline IFT20 & CKO & Severely shortened & Yes & Yes \\
IFT25 & KO & Unaffected & No & No \\
IFT27 & KO & Shortened & No & Mild \\
Gmap210 & KO & Unaffected & No & Mild \\
Bbs8 & MO & Unaffected & Yes & Yes
\end{tabular}

$\mathrm{KO}$, knockout; $\mathrm{CKO}$, conditional $\mathrm{KO}$; SCB, stereocilia bundles. 
each bundle, and the extent of bundle convexity (supplementary material Fig. S2B,C). Although the mean values for these metrics were unchanged, significantly greater variation in bundle convexity was observed in the absence of $B b s 8$. This is consistent with a role for Bbs8 in the specification of the shape, but not the overall size, of the stereociliary bundle.
As uniform orientation of stereociliary bundles is thought to be required for normal hearing, we sought to determine whether the bundle and kinociliary defects observed in $B b s 8^{-/-}$mice lead to deficits in auditory function. Hearing was assessed by measuring auditory brainstem response (ABR) thresholds between 4 and $24 \mathrm{kHz}$ in 2- to 3-month-old mice. Surprisingly, no significant threshold
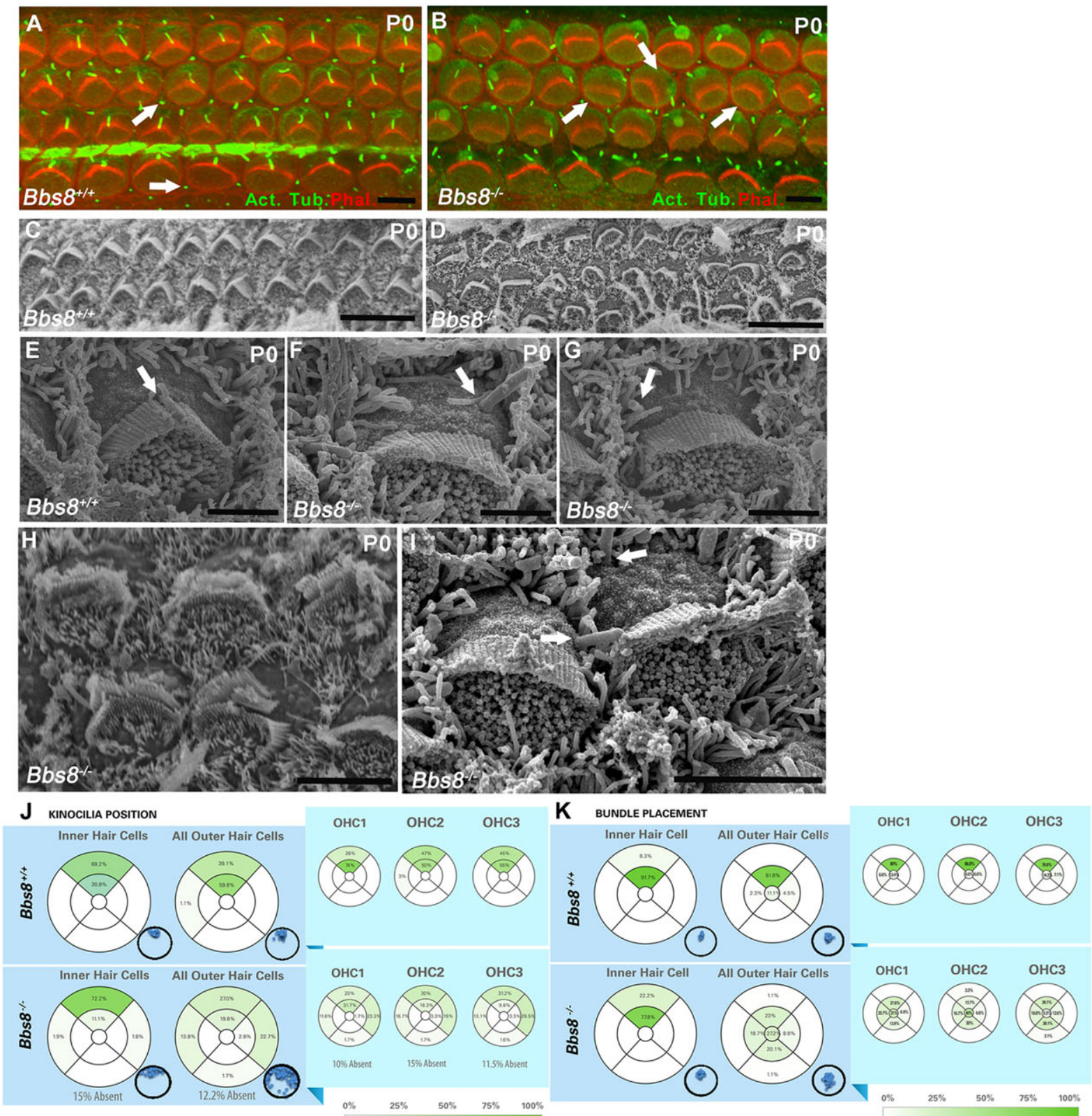

Fig. 2. PCP defects in $B b s 8^{-1-}$ cochleae at PO. (A,B) Whole-mount images of basal cochlear turns from WT (A) and $B b s 8^{-/-}$mutant (B). Filamentous actin (red), acetylated tubulin (green). In WT, chevron-shaped stereociliary bundles uniformly orient towards the lateral edge of each hair cell (upper edge of image). Hair cells have a single kinocilium located at the vertex of the bundle. Single cilia are also present on supporting cells (arrows in A). Stereociliary bundles in $B b s 8^{-1}$ cochleae are variably rotated, flattened and/or mislocalized. Kinocilia are mislocalized or axonemes missing (arrows). (C-I) SEM of basal cochlear turns. Bbs $8^{+/+}$ $(\mathrm{C}, \mathrm{E})$ or $\mathrm{Bbs}^{-{ }^{--}}(\mathrm{D}, \mathrm{F}-\mathrm{I})$ at PO. Low magnification views $(\mathrm{C}, \mathrm{D})$ show overall disruption of bundle polarity in OHCs in $\mathrm{Bbs} 8^{-{ }^{--}}$compared with the uniform alignment in $B b s 8^{+/+}$. (E-I) Higher magnification of stereociliary bundles and kinocilia in $B b s 8^{+/+}$and $B b s 8^{-/-}$OHCs. Note separation between kinocilia and stereociliary bundles in $\mathrm{F}, \mathrm{G}$ and I (arrows) and flattened appearance of many bundles. (J,K) Quantification of kinocilia and bundle positions in $B b s 8^{+/+}$and $B b s 8^{-/-}$

mutant cochleae (PO basal turn). Blue panels show data from IHCs and from all three rows of OHCs combined. Turquoise panels divide bundle and kinocilia positions based on $\mathrm{OHC}$ row. $(\mathrm{J})$ White circles depict lumenal surface of a hair cell, with frequency of kinocilia location in each section indicated as a percentage of total. Inset: actual kinocilium positions. Kinocilia on $\mathrm{Bbs}^{-1-}$ hair cells show minor deviations from control; kinocilia from OHC are broadly distributed around the $\mathrm{OHC}$ edge. All three rows of $\mathrm{OHC}$ in $\mathrm{Bbs}^{-1-}$ cochleae show similar levels of disruption. (K) Position of the bundle center in IHCs and OHCs (see Materials and Methods for details). Inset: actual positions of stereociliary bundle centers. The bias of bundle location towards the lateral side of each hair cell is lost in OHCs from $\mathrm{Bbs}^{-1-}$ cochleae but appears to be maintained in IHCs. Scale bars: $5 \mu \mathrm{m}$ in A,B; $10 \mu \mathrm{m}$ in C,D; $2.5 \mu \mathrm{m}$ in E-G; $5 \mu \mathrm{m}$ in H,I. 
A

ABR thresholds Bbs8 mice

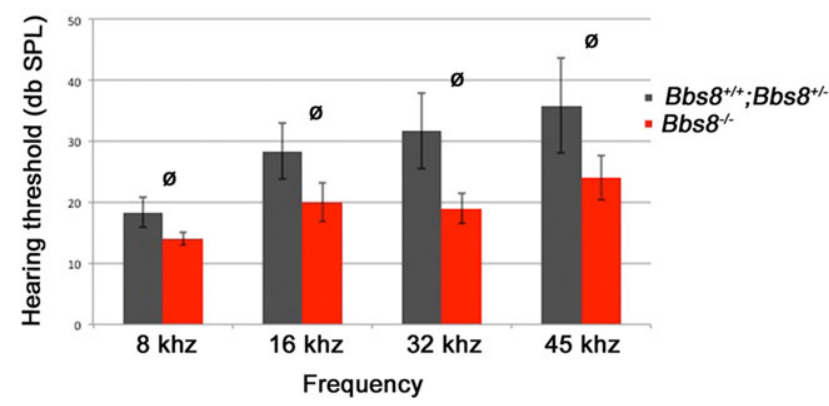

B

Dp grams from Bbs8 mice

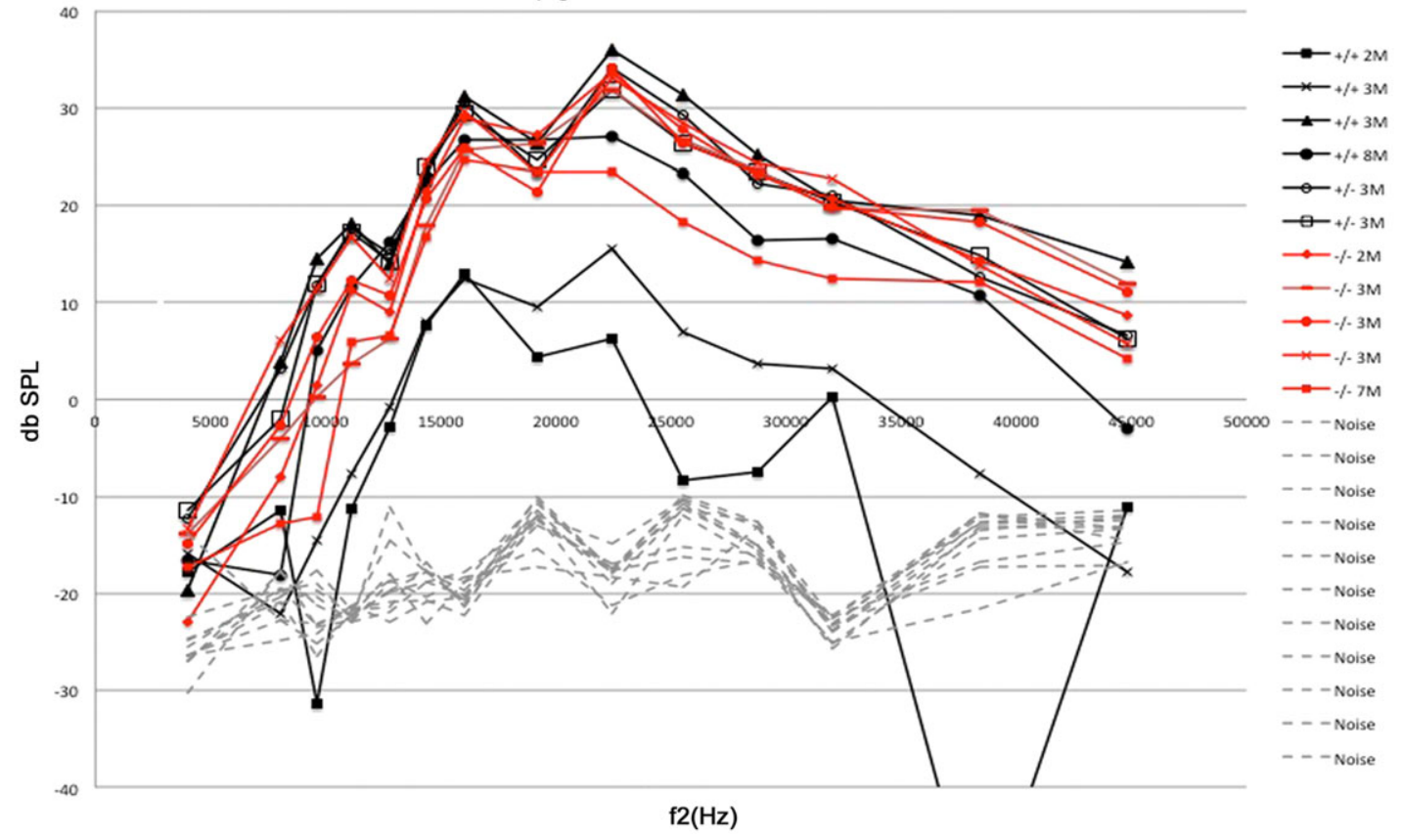

Fig. 3. Analysis of auditory function in $B b s 8^{-1-}$ mice. (A) Auditory brainstem response in control and $B b s 8^{-1-}$ mice tested at indicated frequencies. No significant differences were observed between $B b s 8^{-l-}$ mice and control ( $n=5-6$ per group). Two-way comparisons were carried out using non-parametric, unpaired $t$-tests (Mann-Whitney). (ø) non-significant. Error bars indicate s.d. (B) Distortion-product otoacoustic emission (DPOAE) recordings in $B b s 8^{-1-}$ mice and littermate controls. No differences were observed.

elevations were observed (supplementary material Fig. S2D). As high frequency hearing often shows a greater susceptibility to systemic perturbations, we also examined hearing thresholds up to $45 \mathrm{kHz}$ on a subset of the $B b s 8^{-1-}$ mutants. Even at these higher frequencies, $B b s 8^{-1-}$ mice did not have significantly elevated threshold shifts compared with controls (Fig. 3A). Measurable distortion-product otoacoustic emissions, a measurement of $\mathrm{OHC}$ function, also did not differ between $B b s 8^{-/-}$mutants and controls (Fig. 3B). The basis for the lack of an auditory phenotype is unclear. One possibility, corrective reorientation of bundles, as has been reported for Vangl2 CKOs mutants (Copley et al., 2013), was examined in 8-month-old mice. However, some bundle abnormalities were still present (data not shown).

\section{Bbs8 $^{-1-}$ mutants have PCP defects in other ciliated epithelia}

These results are consistent with a role for Bbs8 in cochlear stereociliary bundle orientation, but not in the convergent extension movements that have been proposed to underlie cochlear extension. As bundle polarity and cochlear extension are often both disrupted in PCP mutants, we sought to determine whether Bbs8 similarly regulated
PCP in two populations of polarized cells that do not undergo convergent extension: vestibular hair cells of the utricular maculae and ependymal cells lining the ventricular system of the brain.

The utricular sensory epithelium comprises a relatively uniform distribution of hair cells arranged in a fan-like shape with stereociliary bundles uniformly oriented along its radial axes (spines). In addition, a line of reversal is present near the mid-point along the medial-tolateral axis such that bundles on either side of the line are oriented towards the center. At P0, the uniform orientation of bundles can be visualized based on labeling of stereocilia with phalloidin (Fig. 4A). By contrast, bundle orientation in $B b s 8^{-/-}$utricles appears irregular, resulting in whorls and disheveled-looking patches (Fig. 4B, arrows). Although these whorls are frequently seen in $B b s 8$ mutants and rarely in the controls, the orientation of individual hair bundles was examined to rule out possible disruptions in bundle structure during dissection or tissue preparation. To quantify this finding, the orientation of stereociliary bundles relative to neighboring hair cells was measured based upon $\beta 2$-spectrin expression (Fig. 4C). $\beta 2$-spectrin is a component of the cuticular plate that anchors the stereocilia and is absent from the fonticulus region at the base of 

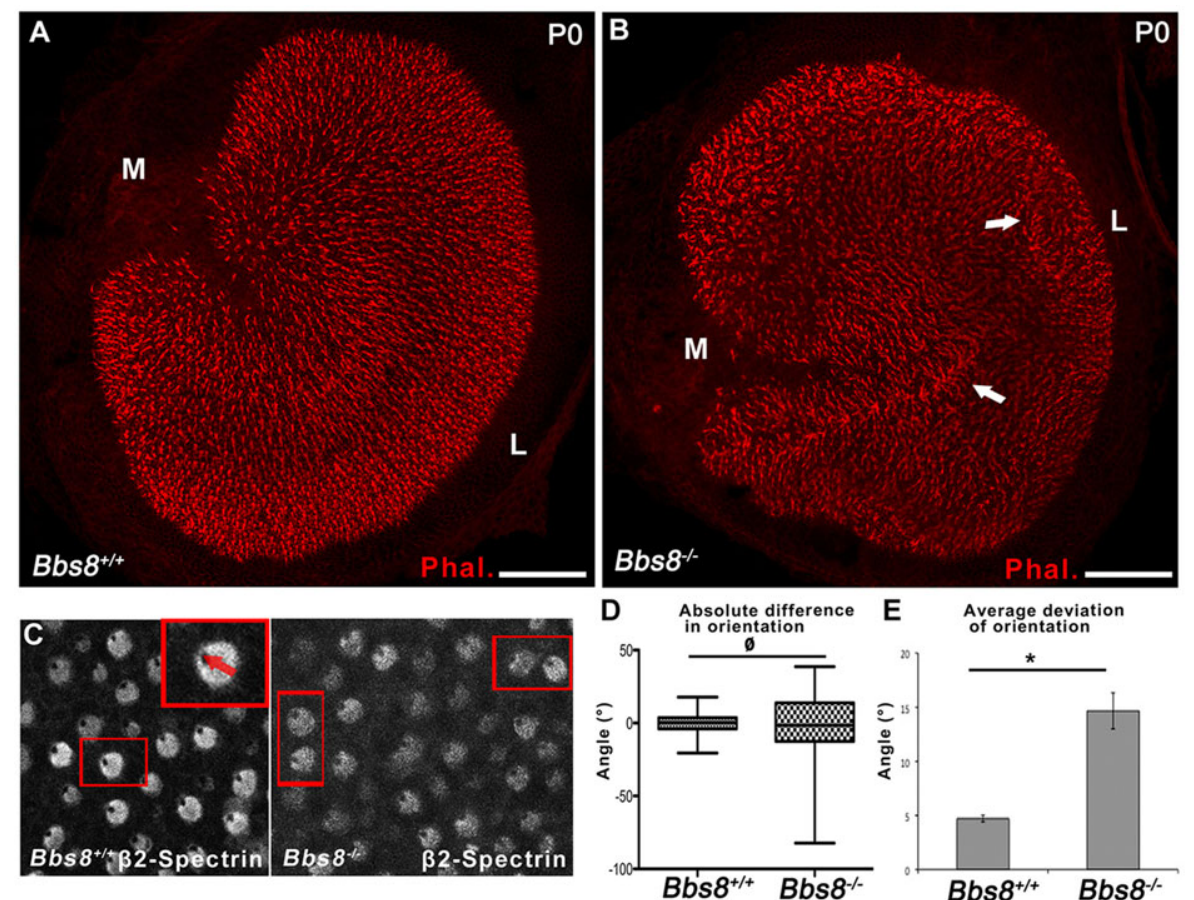

Average deviation
of orientation
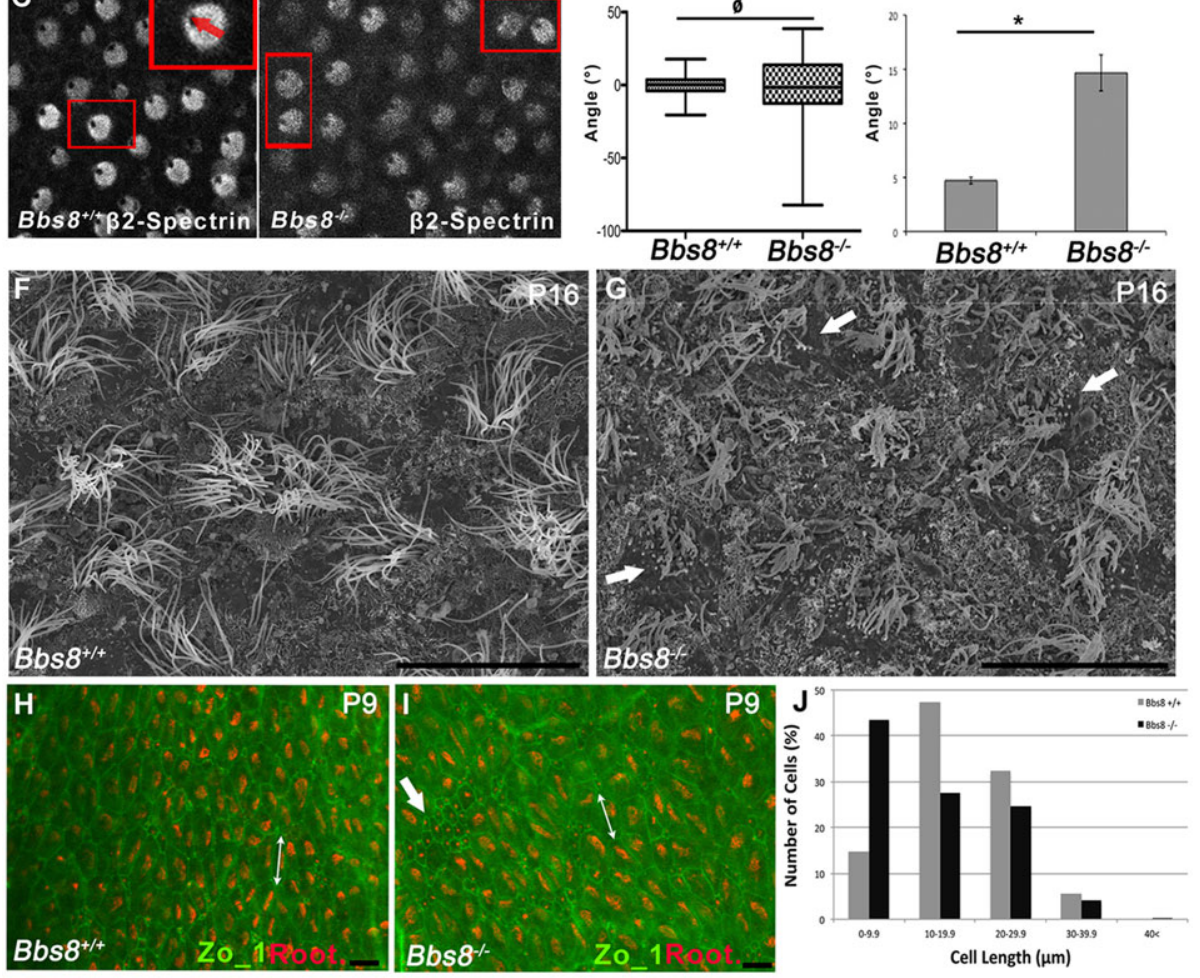

Fig. 4. Additional PCP defects in $B b s 8^{-1-}$ mice. $(A, B)$ Whole-mount utricles from $B b s 8^{+/+}$and $\mathrm{Bbs}^{-1-}$ mutants at P0 stained with phalloidin (red) illustrate stereociliary bundles. Note the uniform bundle orientation along the mediolateral axis in the $\mathrm{Bbs}^{+/+}(\mathrm{A})$. Stereociliary bundles in the $\mathrm{Bbs}^{-/-}$mutant appear disorganized (arrows in B). (C-E) Quantification of utricular polarity. (C) Immunostaining for $\beta$-spectrin highlights orientation of individual cells based on the position of the fonticulus ( $\beta$-spectrin-negative, see inset).

(D) Box plot displaying orientation differences between neighboring hair cells [cluster of $25 ; n=3$ clusters per genotype; median with upper and lower quartiles (box), minimum and maximum values (whiskers)]. (E) Averaged absolute angular deviations calculated in D. Non-parametric, unpaired $t$-tests (Mann-Whitney); (ø) nonsignificant; ${ }^{*} P<0.05$; error bars indicate s.d. $(F, G)$ SEM of brain ventricles (P16) showing ependymal cilia in $B b s 8^{+/+}$and $B b s 8^{-/-}$mice. Short and disorganized ependymal cilia in $B b s 8^{-/-}$ventricles (G, white arrows).

$(\mathrm{H}, \mathrm{I})$ Ventricle ependymal epithelium from $\mathrm{Bbs}^{+/+}$ and $\mathrm{Bbs}^{-1-}$ mice at P9. ZO1 labels cell boundaries (green), rootletin labels cilia rootlets (red). In the mutant, cells with a single spot of rootletin as opposed to a 'mat' of rootletin can be identified (thick arrow). Cell lengths are shown by double-headed arrows. (J) Quantification of cell length in $\mathrm{H}, \mathrm{I}$. Note increased frequency of shorter cells in the $B b s 8^{-1-}$ mutant. Scale bars: $100 \mu \mathrm{m}$ in $\mathrm{A}, \mathrm{B} ; 20 \mu \mathrm{m}$ in $\mathrm{F}, \mathrm{G} ; 10 \mu \mathrm{m}$ in $\mathrm{H}, \mathrm{I}$. the kinocilium. As a result, $\beta 2$-spectrin reveals the location of the kinocilium on each cell. To determine whether absence of Bbs8 leads to changes in polarization, the average difference in the angle of polarization between one hair cell and its neighbors was determined for both control and $B b s 8^{-/-}$utricles (see Materials and Methods for details). Whereas the average angular difference was unchanged between mutants and controls, a statistically significant difference in standard deviation was measured between the two groups (Fig. 4D,E). The line of reversal was also more difficult to identify in $B b s 8^{-/}$ mutant utricles. These results are consistent with a disruption in bundle polarization.

PCP signaling has also been shown to play a role in the development of ependymal cilia, with defects leading to hydrocephalus (Tissir et al., 2010). Polarization of ependymal cells is tightly correlated with maturation and differentiation of multi-ciliated cellular morphologies. Consistent with this, $B b s 8^{-/-}$ mice are born at Mendelian ratios yet are underrepresented at weaning and display hydrocephaly (data not shown). SEM of ependymal cells at P16 indicated severely stunted cilia in $B b s 8^{-/-}$ ventricles (Fig. 4F,G). This phenotype was reminiscent of other PCP mutants in which ependymal cilia became basally embedded, instead of presenting normally on the apical surface (Tissir et al., 2010). Immunohistochemical analysis of the lumenal surface (length) at earlier time points (P9) suggests that ependymal cell maturation is compromised upon loss of Bbs8 (Fig. 4H,I, double arrow). There was also a wider variation in cell length in $B b s 8^{-/-}$ tissue (Fig. 4J), which might have been caused by a failure of cells to polarize. Finally, undifferentiated cells with only a single spot of rootletin (suggesting a single primary cilium) were more commonly observed in mutant tissue (Fig. 4I, white arrow).

\section{Ift20 regulates $P C P$ in the cochlea}

PCP phenotypes were also observed in cochleae from animals in which Ift 20 was deleted from the inner ear by crossing Ift $20^{f l o x}$ flox mice with FoxG1 ${ }^{\text {cre }}$ (referred to as Ift20 $0^{\text {cko/cko; }}$, see Materials and Methods for details). Conditional mutants are not viable, and 

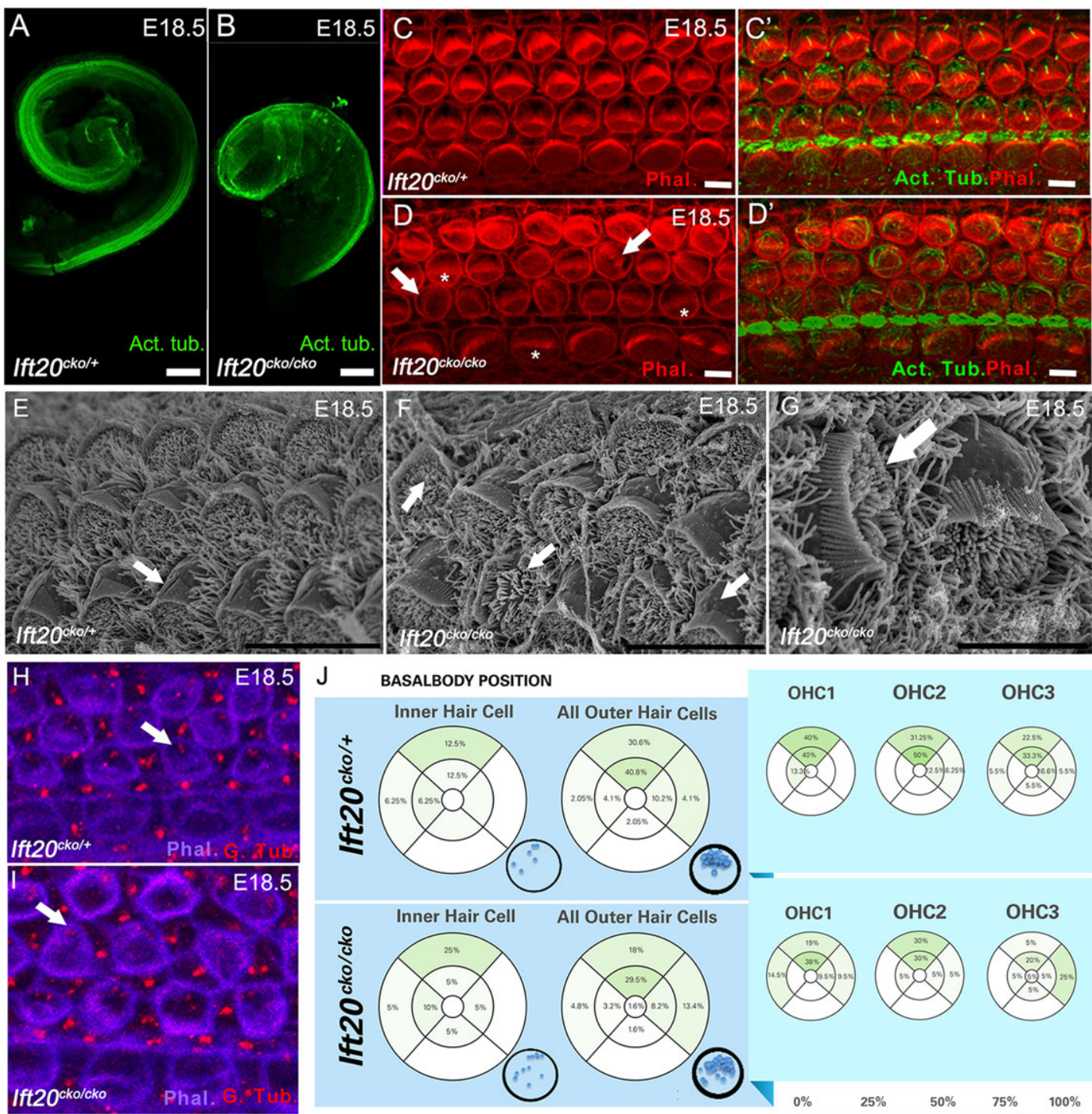

Fig. 5. PCP defects in Ift20 ${ }^{\text {ckolcko }}$ cochleae. (A,B) Cochlea extension defects in Ift20 ${ }^{\text {ckolcko }}$ cochleae. Dissected cochlear ducts at E18.5 stained with acetylated tubulin reveals marked shortening of Ift $20^{\text {ckolcko }}$ cochlea. (C,D) Basal turn of the organ of Corti from Ift20 $0^{\text {ckol+ }}$ and Ift2 $0^{\text {ckolcko }}$. Filamentous actin (red) and acetylated tubulin (green) are labeled. (C) In Ift20 cko/t mice, stereociliary bundles are uniformly oriented with a kinocilium (green) located at the vertex of each bundle. (D) In Ift20 ckolcko cochleae, kinocilia and other cilia are absent and stereocilia bundles are flattened (asterisks) and rotated (white arrows). Internal microtubules are still present. (E-G) SEM of basal turn organ of the Corti from Ift20 ${ }^{c k o /+}(E)$ and Ift20 ${ }^{c k o / c k o}(F, G)$ cochleae $(E 18.5)$. In Ift20 ${ }^{c k o l+}$ cochleae, each uniformly aligned stereociliary bundle has a kinocilium at the vertex (small arrow). In Ift $20^{\text {ckolcko }}$ cochleae, kinocilia are absent and bundle morphology is disrupted (small arrows in F). Cells with 'circular' stereocilia bundles (large arrow) are also observed. (H-J) Quantification of basal body position in hair cells from the basal turn of $\mathrm{Itt2} \mathrm{O}^{\mathrm{ckol}+}$ and Ift2 $\mathrm{O}^{\text {ckolcko }}$ cochleae at $\mathrm{E} 18.5$. (H,I) Because kinocilia are absent, basal bodies were visualized by labeling for $\gamma$-tubulin (red; white arrows). Cell boundaries are indicated by F-actin (purple). Stereociliary bundles are not seen because the focal plane is below the lumenal surface of the epithelium. (J) Summary of changes in basal body positioning in Ift2 $0^{c k o / c k o}$ cochleae. Blue panels show data from IHCs and from all three rows of OHCs combined. Turquoise panels divide basal body positions based on $\mathrm{OHC}$ row. Percentage positioned in each section of inner and OHCs. A large number of basal bodies from IHCs could not be visualized due to technical reasons. Inset: overlay of actual basal body positions. Basal bodies were positioned more centrally compared with control, in which they had begun to migrate to the abneural edge by this age. Scale bars: $100 \mu \mathrm{m}$ in A,B; $5 \mu \mathrm{m}$ in C,D; $10 \mu \mathrm{m}$ in E,F; $5 \mu \mathrm{m}$ in G.

analyses were therefore performed at E18.5. Cochlear ducts from Ift $20^{\text {cko/cko }}$ mice were significantly shorter than in littermate controls (Fig. 5A,B; supplementary material Fig. S3A) and showed a marked broadening of the sensory epithelia in the apex (supplementary material Fig. S3B,C). Ift20 is required for ciliogenesis, thus, as expected, kinocilia were absent in Ift $20^{\text {cko/cko }}$ hair cells (Fig. 5C-G), which also served to confirm deletion of Ift20. Hair cells exhibited flattened and rotated bundles, similar to those observed in $B b s 8^{-/-}$ mutant cochleae and other ciliary mutants that lack kinocilia (Fig. 5C-G) (Jones et al., 2008; Sipe and Lu, 2011). SEM confirmed these findings (Fig. 5E-G). As observed in other mutants lacking kinocilia, hair cells with circular stereociliary bundles could be identified, albeit infrequently (Fig. 5G, white arrow). As ciliary axonemes were missing in Ift $20^{\text {cko/cko }}$ hair cells, changes in polarization were quantified based on the position of the basal body (Fig. 5H-J). In hair cells from control cochleae, the majority of basal bodies were located in the lateral quadrant (Fig. $5 \mathrm{H}$, white arrow). By contrast, OHCs from Ift $20^{\text {cko/cko }}$ cochleae often lacked basal bodies, and those that could be identified were more widely distributed across the lumenal surface (Fig. 5J). Analysis of basal body locations by row suggested that the first and third row OHCs show greater defects in the absence of Ift20 (Fig. 5J). Absent basal 

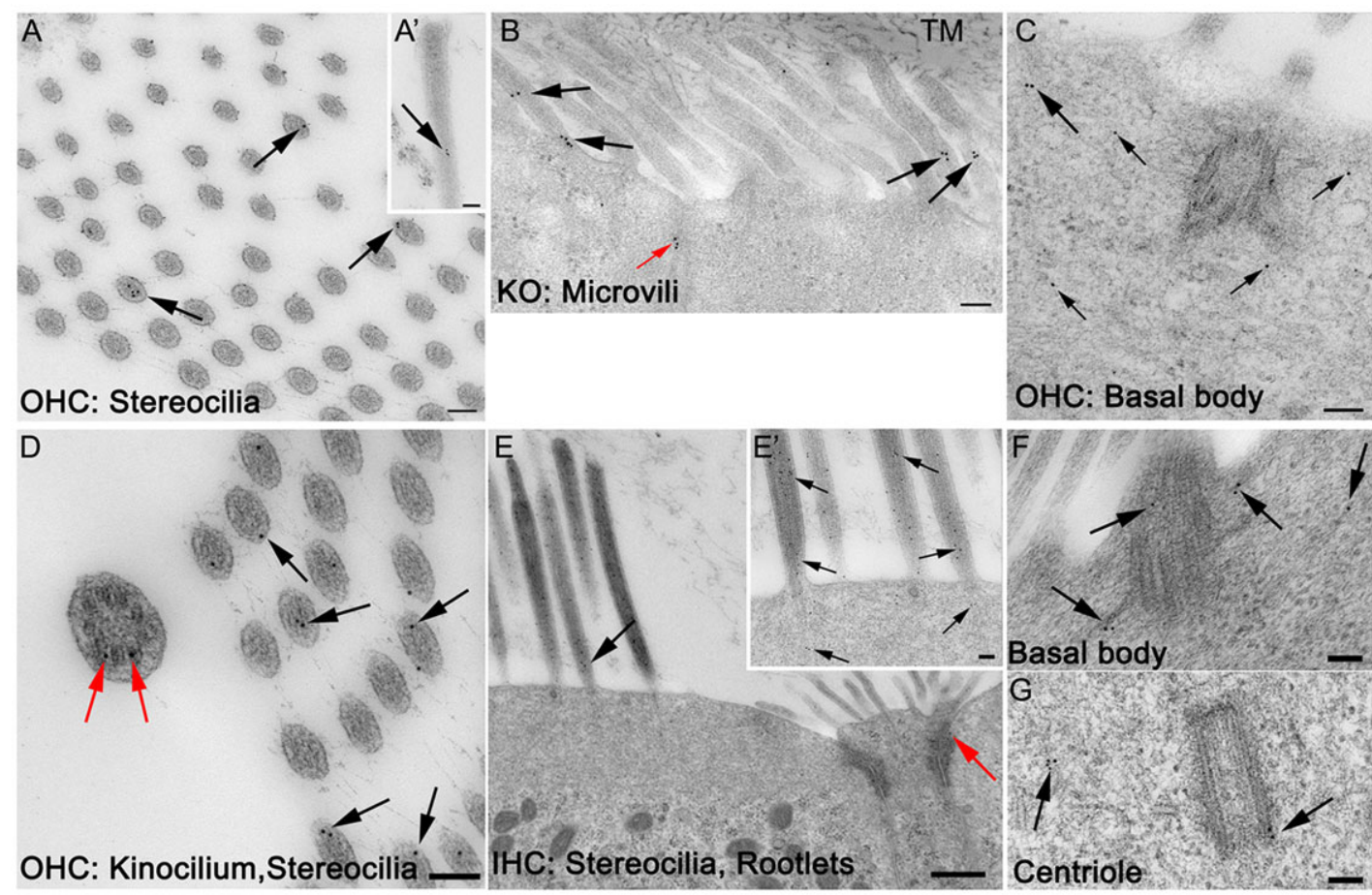

Fig. 6. Immunogold localization of Bbs2 and Ift20 in P0 cochleae. (A-C) Bbs2 (black arrows) localizes to actin-rich structures such as OHC stereocilia illustrated in horizontal $(A)$ and vertical section $\left(A^{\prime}\right)$. Similarly, Bbs2 is present in microvilli on epithelial cells within Köllicker's organ (B). Also, note the cluster of Bbs2 labeling on a vertical tract of microfilaments contacting the apical surface (red arrow). These microfilaments are similar in structure to those in microvilli, and are probably actin. Small dark specks in the tectorial membrane (TM) are cross-sections of filaments, not labeling for Bbs2. (C) Bbs2 (arrows) is also localized near the basal body in OHCs. (D-G) Localization of Ift20. In a horizontal section through an OHC stereociliary bundle, Ift20 is present in both the kinocilium (red arrows) and stereocilia (black arrows). (E) Similar Ift20 localization is present in vertical cross-sections (lengthwise) through an IHC bundle. Note in $\mathrm{E}^{\prime}$ (IHC stereocilia) that labeling continues into the rootlets but is not common among the surrounding actin filaments of the cuticular plate. Labeling at the electron-dense cell junctions (red arrow), where membrane trafficking is concentrated, is also observed. Ift20 is also associated with basal bodies (in an inner border-supporting cell; F) and associated centrioles (OHC in G). OHC, outer hair cell; IHC, inner hair cell; KO, Köllicker's organ; TM, tectorial membrane. Scale bars: $100 \mathrm{~nm}$.

bodies might be a result of deeper positioning within the hair cells but could also reflect technical difficulties with the antibody reaction. By contrast, basal body locations on IHCs appeared comparable between control and Ift20 cko/cko (Fig. 5H-J).

\section{Localization of ciliary proteins to actin-rich structures}

Previous studies have suggested that Bbs and Ift proteins are not strictly associated with cilia and have shown localization to actin-rich cellular regions. Localization to actin-based structures might be significant in hair cells because the actin-rich stereocilia develop adjacent to the tubulin-based kinocilium, and molecules functioning in either structure could act to polarize the bundle. To determine where Bbs and Ift proteins localize within cochlear hair cells, immunogold labeling/TEM analysis was performed on cochleae at P0. Existing antibodies directed against Bbs8 proved unsuitable for TEM. However, as Bbs8 and Bbs2 are both components of the BBSome complex required for cilia biogenesis (Nachury et al., 2007), an antibody against Bbs2 was used as a proxy for Bbs8. Bbs2 localized to actin-rich structures in developing hair cells with a particular concentration along stereocilia and microvilli (Fig. 6A-C). Of particular interest was clustering of $\mathrm{Bbs} 2$ on vertical tracts of microfilaments contacting the apical surface (Fig. 6B). These microfilaments were similar in structure to filaments within microvilli, and are therefore presumably actin based. Similar actin-associated localization patterns for Bbs2 were seen in additional ciliated epithelia in the ventricular zone and choroid plexus (supplementary material Fig. S4). Ift20 labeling could be seen in the kinocilium (Fig. 6D, red arrows) and was also abundant in actin-rich microvilli (data not shown) and stereocilia of OHCs and IHCs (Fig. 6D-E'). Labeling was also observed near basal bodies (Fig. 6F) and associated centrioles (Fig. 6G).

\section{Absence of Bbs8 or Ift20 leads to a lack of Vangl2 accumulation at the hair cell/support cell membrane}

These results demonstrate roles for Bbs8 and Ift20 in bundle polarization; however, the specific effects of these molecules are unclear. Previous studies have placed other cilia-related proteins, such as Ift88, downstream of the core PCP factors, by demonstrating that asymmetric localization of core PCP proteins occurs normally in these mutants. To determine whether a similar localization of core PCP proteins occurs in $B b s 8$ or Ift20 mutants, membrane localization of Vangl2 was determined by immunocytochemistry at P0 and E18.5, respectively (Fig. 7A-D). In control cochleae (Fig. 7A,C), Vangl2 was localized along both medial and lateral surfaces of pillar cells (Fig. 7A,C, white asterisk), and asymmetrically at junctions between medial hair cell and lateral support cell surfaces (Fig. 7A,C, white arrow). By contrast, in $B b s 8$ mutants, although Vangl2 was still detected along pillar cells, membrane accumulation at hair cell-support cell junctions appeared reduced (Fig. 7A,B). Similar changes were observed in Ift $20^{\text {cko/cko }}$ cochleae, although overall membrane localization appeared somewhat more reduced (Fig. 7C,D). This result contrasts with localization in other cilia mutants, in which Vang12 was shown to be unaltered, including Mkks, Ift27, Ift25, Gmap210 and Ift88 (supplementary material Fig. S5) (Jones et al., 2008). In order to determine whether the decreased labeling of Vangl2 in Bbs 8 mutants was a result of decreased protein abundance or changes in membrane 

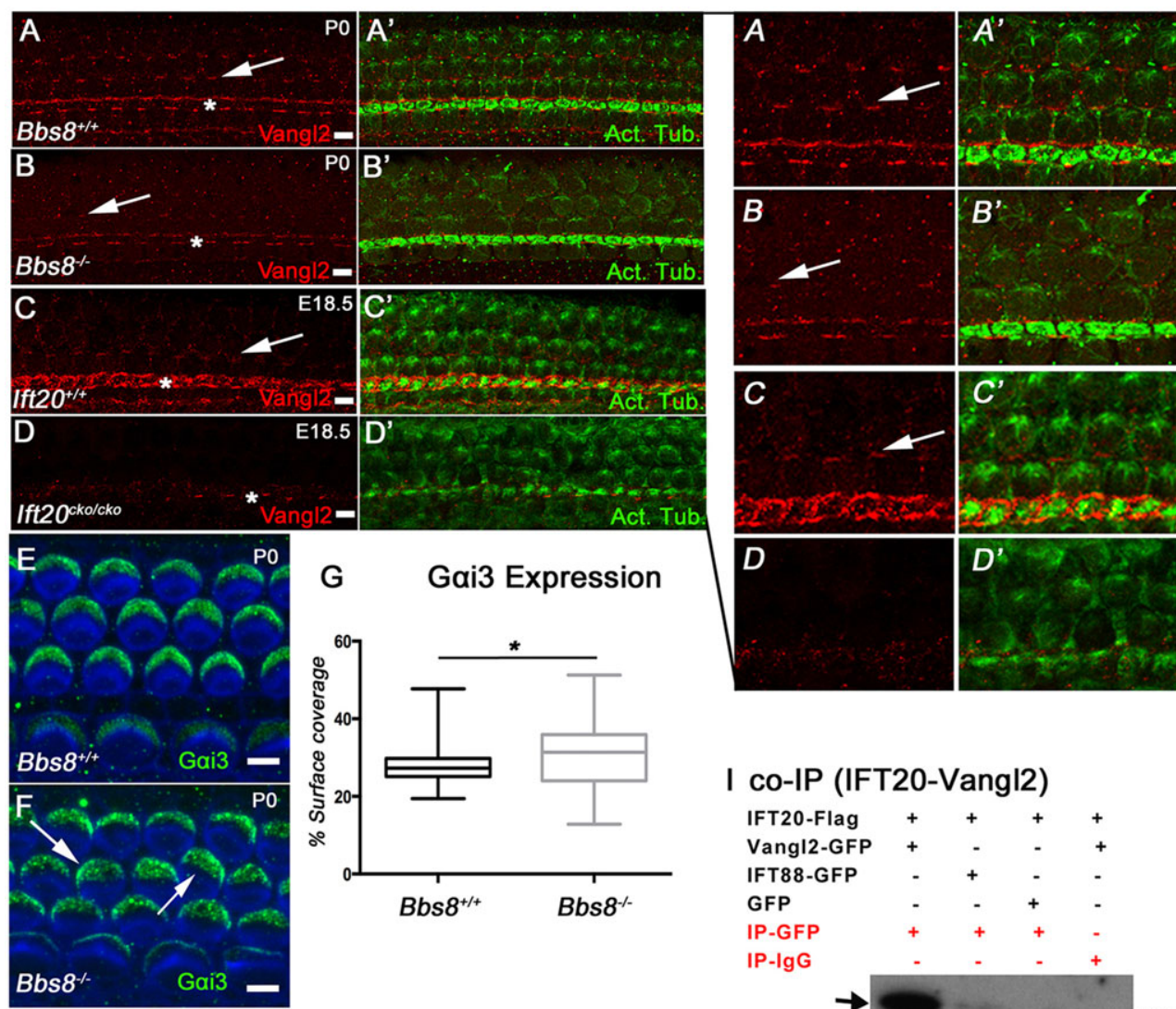

\section{G Gai3 Expression}
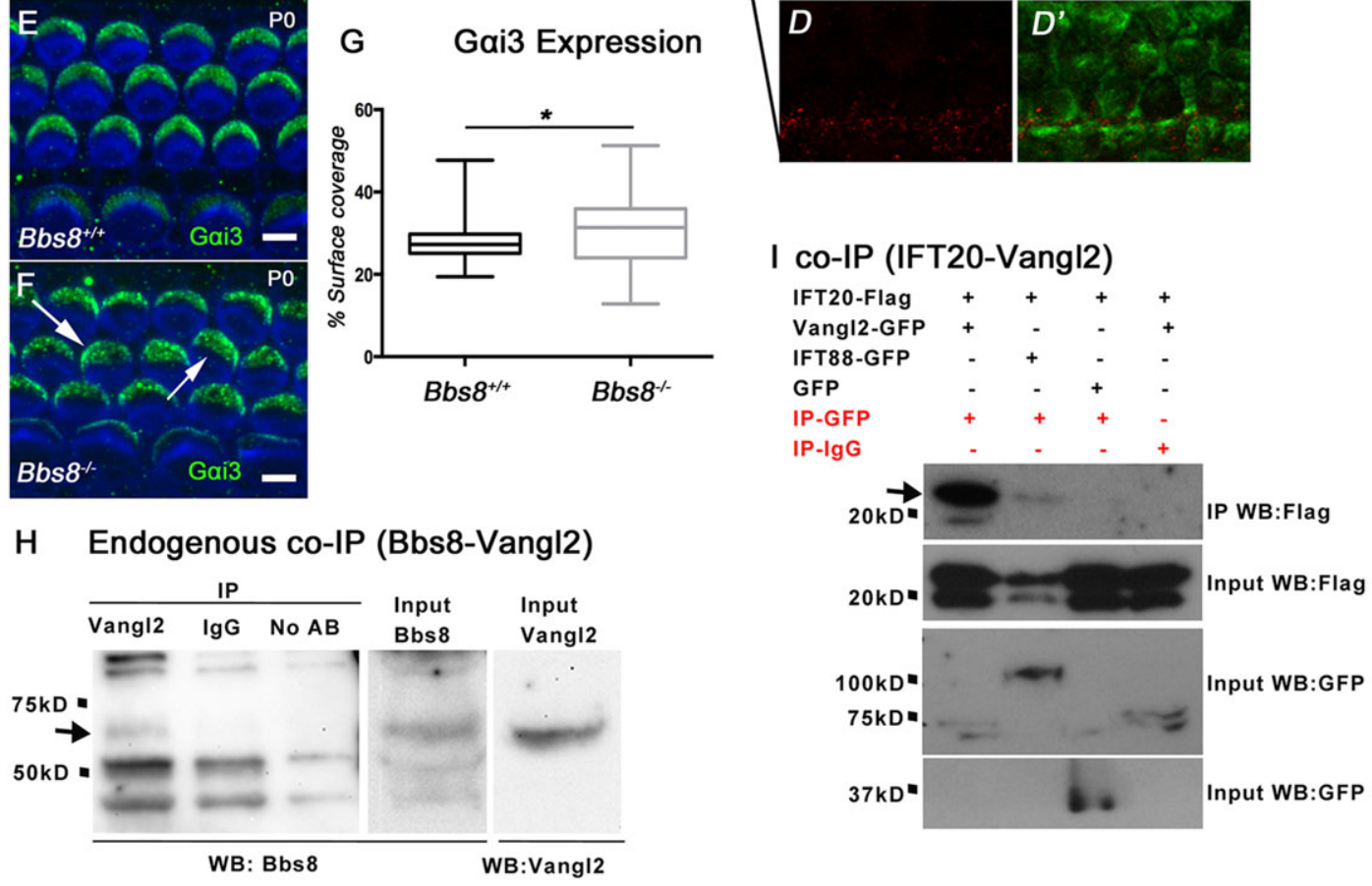

Fig. 7. Vangl2 localization is dependent on Bbs8 and IFT20. (A-D) Immunolocalization of Vangl2 (red) and $\alpha$-acetylated tubulin in control and $B b s 8^{-1-}$ and Ift20 ${ }^{\text {ckolcko }}$ mutant cochleae in the basal turn at P0 (Bbs8) or E18.5 (Ift20). (A,C) In controls, Vangl2 localizes along pillar cells (white asterisks) and at junctions between lateral supporting cells and medial hair cell edges (white arrows). (B,D) By contrast, in both $B b s 8^{-I-}$ and lft2 $\mathrm{O}^{\text {ckolcko }}$ cochleae, Vangl2 membrane localization is reduced, with some localization remaining along the pillar cells (white asterisks). Disrupted Vangl2 localization appears greater in Ift20 ckolcko . $(\mathrm{E}, \mathrm{F})$ Expansion of Goi3 (green) domain in $B b s 8^{-1-}$ cochlea (PO) (white arrows). Hair cells counterstained with phalloidin (blue), outlining cell borders and stereociliary bundles. (G) Box plot of Goi3 expansion. Non-parametric, unpaired $t$-tests (Mann-Whitney); ${ }^{*}<<0.05 ; n=3$ samples per genotype and 15 cells per sample; median with upper and lower quartiles (box), minimum and maximum values (whiskers). (H) Immunoprecipitation of Bbs8 using an antibody against Vangl2 from brain ventricular tissue (P0). Western blot for Bbs8 (black arrow at $\sim 58 \mathrm{kDa}$ ) shows association between the two proteins. Bbs8 and Vangl2 $(\sim 59 \mathrm{kDa})$ are present in the input. (I) Co-immunoprecipitation (co-IP) of IFT20-FLAG with Vangl2-GFP using an anti-GFP antibody for the IP and an anti-FLAG antibody for the western blot. Empty-GFP and IP with IgG were used as negative controls. IFT88-GFP, a known binding partner of IFT20, was used as positive control. Positive co-IP bands were only observed with Vangl2-GFP and IFT20-Flag and in positive control (black arrow). Note reduced input of IFT20-FLAG in positive control (IFT88) lane. Scale bars: $5 \mu \mathrm{m}$.

targeting, cochlear tissue was separated into cytoplasmic and membrane fractions and then probed for expression of Vangl2 by western blot. No significant difference between control and mutants was observed for either fraction (supplementary material Fig. S6), suggesting that Bbs8/ Ift20 play a role in targeting Vangl2 to specific regions of the cell rather than in regulation or overall membrane localization.

Recent studies identified a G-protein-dependent signaling pathway acting in a cell-autonomous manner, independent of the core PCP proteins, that regulates the migration of the kinocilium and patterns the apical hair cell surface (Ezan et al., 2013). Moreover, localization of GTP-binding protein alpha-i subunit 3 (Goi3), a key factor in this migration, is disrupted in Mkks (Bbs6) mutants. To determine whether Bbs8 plays a similar role, localization of Goi3 was determined in Bbs 8 mutants (Fig. 7E-G). Overall, there is an expansion of the Goi3 domain in the absence of Bbs8, consistent with abnormal hair bundle morphology and kinocilia mislocalization. There also seems to be a larger variation in Goi3 expansion over the surface of the hair cells, as opposed to a more uniform distribution in controls.

The results presented above are consistent with previous studies demonstrating an interaction between Bbs8 and Vangl2 in vitro (May-Simera et al., 2010). To determine whether a similar association exists in vivo, we performed co-immunoprecipitation 
Table 2. Affymetrix microarray data from $\mathrm{Bbs}^{-1-}$ cochlear sensory epithelia

\begin{tabular}{|c|c|c|c|}
\hline Pathway name & Enrichment score & Enrichment $P$-value & Pathway ID \\
\hline Cell adhesion molecules (CAMs) & 12.1192 & $5.45 \mathrm{E}-06$ & kegg_pathway_88 \\
\hline Protein digestion and absorption & 10.7936 & 2.05E-05 & kegg_pathway_140 \\
\hline ECM-receptor interaction & 9.70125 & $6.12 \mathrm{E}-05$ & kegg_pathway_12 \\
\hline Focal adhesion & 9.36983 & $8.53 E-05$ & kegg_pathway_50 \\
\hline Gene symbol & Fold change & $P$-value & RefSeq Transcript ID \\
\hline Dkk3 & -2.01624 & 0.0102556 & NM_015814 \\
\hline Frzb & -2.1041 & 0.0128049 & NM_011356 \\
\hline Fzd7 & -1.64635 & 0.000811933 & NM_008057 \\
\hline Fzd8 & -1.92206 & 0.02508 & NM_008058 \\
\hline Sfrp1 & -2.94657 & 0.00142244 & NM_013834 \\
\hline Wnt5a & -1.60999 & 0.0132632 & NM_001256224 \\
\hline Wnt7a & -2.27966 & 0.0241439 & NM_009527 \\
\hline Wnt9a & -2.32839 & 0.0230976 & NM_139298 \\
\hline
\end{tabular}

Upper table: top four pathways identified using Partex pathway analysis, based on differentially expressed gene transcripts. Bottom table: selection of Wnt transcripts downregulated in $B b s 8^{-1-}$ cochlear sensory epithelia.

(co-IP) pull-down assays from endogenous neuronal tissue using antibodies specific for Vangl2 or Bbs8. Neuronal tissue was used because of the greater relative abundance of both proteins. After IP, using an anti-Vangl2 antibody, Bbs8 was detected by western blot. This band was absent from control IPs (Fig. 7H, black arrow). Next, we examined possible associations of Ift 20 with Vangl2 via co-IP, using tagged constructs transfected into HEK293 cells. We were able to identify Ift20-Flag after IP with Vang12-GFP (Fig. 7I, black arrow). Ift88-GFP was used as a positive control, which also precipitated Ift20-Flag, whereas negative controls showed no bands. Together, these findings suggest that the PCP protein Vangl2 physically interacts with Bbs 8 and Ift20, and that this interaction mediates the asymmetric accumulation of Vangl2 in polarized cells.

\section{Altered gene expression in $\mathbf{B b s}^{-1-}$ mutant cochlea}

To identify pathways that might be altered as a result of the deletion of $B b s 8$, we performed a microarray analysis using cochlear tissue harvested from $\mathrm{P} 0 \mathrm{Bbs} 8^{+/+}$and $B b s 8^{-/-}$mice. After normalization using the RMA algorithm, significantly differentially expressed genes with a twofold change or more $(P<0.05)$ were selected, based on an ANOVA using the Partek Genomics Suite software. The resulting genes were analyzed using the Partek Pathway software, which identifies KEGG Pathways enriched with these genes. Three of the top four pathways with enrichment scores above 9.0 and highly significant $P$-values $(P$-values $<8.6 \mathrm{E}-05)$ were related to cell adhesion, cell migration and cytoskeletal rearrangements (Table 2; supplementary material Table S1). Changes in all of these pathways can be a consequence of disruption in PCP signaling (CarreiraBarbosa et al., 2009; Cui et al., 2013; Ezan and Montcouquiol, 2013; Narimatsu et al., 2009; Saburi and McNeill, 2005), suggesting that the observed phenotypic defects in the $B b s 8^{-1-}$ mice are attributable to its dysregulation. In addition, as previous work has suggested that multiple Wnt pathways can interact in a mutually antagonist fashion, we examined changes in genes directly involved in Wnt signaling. Here, we found downregulation of transcripts encoding many Wnt ligand and Wnt receptor molecules (Table 2).

\section{DISCUSSION}

There is growing evidence supporting an involvement of ciliarelated proteins in regulation of PCP. However, understanding the potential role of cilia in this pathway is complicated due to the redundancy of ciliary and PCP genes. In addition, cell polarity is sensitive to generalized cellular abnormalities, making it difficult in some circumstances to directly link a mutation to PCP-specific deficits. To better understand this, we analyzed polarity defects within the cochleae of mice with targeted mutations in several cilia-related genes. It is remarkable that, although these proteins have similar functions in other systems, we observed distinct cochlear phenotypes for the different mutants. For example, deletion of Gmap 210, Ift 27 or Ift 25 showed only mild cochlear disruptions. By contrast, more severe PCP defects were observed when $B b s 8$ or Ift 20 were deleted, including changes in stereociliary bundle orientation and morphology, and mislocalization of the core PCP transmembrane protein Vangl2. The collective phenotype was greatest in Ift 20 mutants that were missing ciliary axonemes altogether and also showed changes in cochlear duct extension. This phenotype is consistent with other ciliary mutants, such as Ift 88 and Kif3a, in which complete loss of the ciliary axoneme results in shortened cochlear ducts and mis-positioned stereociliary bundles. However, in Ift 88 and Kif $3 a$ mutants, the membrane localization of Vangl2 remains intact (Jones et al., 2008; Sipe and Lu, 2011). This is in contrast with the results presented here for $B b s 8$ and Ift 20 mutants, which suggest that a subset of cilia proteins does affect PCP signaling at the level of PCP protein localization. These results also indicate that the PCP phenotype observed in a subset of ciliary mutants is related to the disruption of PCP signaling. We propose that those ciliary proteins contribute to protein trafficking, thereby regulating the asymmetric localization of PCP molecules.

Many proteins associated with cilia function occur in complexes localized to cilia-related subdomains, such as the basal body, transition zone or ciliary axoneme (van Dam et al., 2013). More recently, some of these proteins have also been observed in other cellular regions, such as dendrites of retinal neurons and epithelial focal adhesions (Finetti et al., 2009; Sedmak and Wolfrum, 2010; Yuan and Sun, 2013). These observations suggest that cilia-related proteins have additional cellular functions away from the cilium, although these functions could still be orchestrated via the basal body in its capacity as a microtubule-organizing center. Given that a primary role for ciliary proteins is the movement of cargo along microtubules, it is highly likely that these proteins might also regulate aspects of intracellular trafficking along the cytoskeleton, such as vesicular transport (Delaval et al., 2011; Kim and Tsiokas, 2011; Robert et al., 2007; Follit et al., 2008, 2006; Pedersen et al., 2008; Sedmak and Wolfrum, 2010). For instance, Ift20 has been shown to contribute to vesicular trafficking from the Golgi to the base of the 
cilium (Follit et al., 2006). These observations, together with the disrupted membrane accumulation of Vangl2 in Ift20 mutants, indicate that Ift20 is required for the delivery of Vang12-containing vesicles to the cell surface. These observations also suggest that other members of the IftB complex contribute to Vangl2 targeting. However, at least two members of that complex, Ift25 and Ift27, do not appear to play crucial roles, as deletion of either gene leads to only mild cochlear PCP defects. This suggests a unique requirement for Ift20 compared with other Ifts, as well as potential novel roles for this protein that might be independent of its association with Ift complexes. A similar result was observed in $B b s 8$ mutants, a finding that is consistent with the direct physical interactions between Bbs8 and Vangl2 [May-Simera et al. (2010) and this study]. Like Ift20, protein-localization studies have expanded the potential functions for Bbs proteins beyond the cilia. In the cochlea, Bbs2, 4 and 6 display a range of non-cilia-related cellular distributions, including in the vicinity of cellular membranes and actin-rich regions (May-Simera et al., 2009). In cultured cells, Bbs proteins are localized to actin-rich focal adhesions, where they negatively modulate the actin cytoskeleton (Hernandez-Hernandez et al., 2013). Similarly, we found Bbs 2 enrichment in the actin-based stereocilia of hair cells. Together, these observations suggest that Ift 20 and Bbs 8 can function independently of the ciliary axoneme, and might traffic proteins along microtubules or filamentous actin to cellular locations other than cilia.

The basis for the lack of an auditory phenotype in $B b s 8$ mutants is unclear. One possibility is that there is a corrective reorientation of bundles, as has been reported for Vangl2 CKO mutants (Copley et al., 2013); however, our preliminary evidence suggests that this is not the case. Other cilia mutants, such as Mkks (Bbs6) and Alms1, have milder bundle disruptions than $B b s 8$ mutants, yet display more severe auditory phenotypes (Jagger et al., 2011; Ross et al., 2005). This suggests that auditory dysfunction in cilia mutants is not necessarily directly linked to alterations in stereociliary bundle morphology or mechanotransduction, further arguing that these genes encode additional intracellular functions independent of cilia. Based on these results, we propose that a subset of proteins that had originally been identified by their association with cilia might in fact function in broader roles related to the intracellular trafficking of membrane-bound proteins throughout the cell, although these functions might still be organized via the basal body. Bbs 8 and Ift20 appear to be members of this group in that they act upstream of cilia localization by targeting Vangl2 (and other) PCP proteins to the membrane. Although this does not rule out a role for Bbs8 and Ift20 in ciliary migration, it seems clear that other cilia proteins, such as Ift88, Mkks and Kif3a, have more restricted functions and only contribute to ciliary migration.

One important function attributed to the primary cilium is regulating a transition between the canonical ( $\beta$-catenin dependent) and non-canonical (PCP) Wnt signaling pathways (Simons et al., 2005). As canonical Wnt signaling regulates transcription (Dickinson and McMahon, 1992), we tested whether loss of Bbs8 might also affect gene expression. Indeed, microarray data from $B b s 8^{-/-}$cochleae show changes in gene expression. Many of these genes encode proteins involved in Wnt signaling and downstream effectors, suggesting additional consequences of $B b s 8$ deletion besides PCP signaling. As a result, loss of ciliary function might upset the delicate balance between canonical and non-canonical Wnt signaling. By contrast, other studies have suggested no connection between cilia and Wnt signaling. For example, Ift 88 zebrafish mutants lack all cilia, but have normal canonical and noncanonical Wnt signaling (Borovina and Ciruna, 2013; Huang and Schier, 2009), as do Ift88, Ift172 and Kif3a mutant mouse embryos
(Ocbina et al., 2009). These differences might reflect species-, tissue- or time-dependent differences in ciliary contributions towards Wnt signaling. Moreover, normal Wnt responsiveness might be retained if the basal bodies, and therefore trafficking to the basal body, remain intact in these mutants.

Our results, in combination with previous reports on the effects of deletion of $B b s 8$ (Tadenev et al., 2011), suggest a greater role for Bbs 8 in PCP by comparison to other Bbs proteins (Ross et al., $2005)$. If this is the case, then patients with mutations in $B B S 8$ should display an increased prevalence of 'PCP-like' phenotypes. Indeed, $B B S 8$ was first identified in a family with Bardet-Biedl syndrome, in which a homozygous null mutation results in randomization of left-right body axis symmetry, another phenotype possibly related to PCP defects (Ansley et al., 2003; Aw and Levin, 2009). Several BBS patients with deleterious mutations in $B B S 8$ have been identified, who in addition to the typical disease phenotypes (obesity, retinopathy, polydactyly) harbor additional clinical manifestations including renal cystic disease, shortened limbs, hearing impairment, dilated ventricles and situs inverses (P. Beales, personal communication), features that could be suggestive of perturbed Wnt signaling culminating in aberrant PCP. Altogether, our results and these observations are consistent with the role of ciliary proteins extending beyond the cilia and basal body. Clearly, a more complete understanding of the role of 'ciliary' proteins in cellular signaling pathways and other biological phenomena is crucial for our understanding of cellular and developmental biology, as well as for the development of targeted treatment strategies.

\section{MATERIALS AND METHODS}

\section{Mice}

Animal care and use was in accordance with NIH guidelines and conformed to institutional ACUC regulations. Generation and genotyping of $\mathrm{Bbs} \mathrm{8}^{-/-}$ and Ift $20^{\text {flox/flox }}$ mutants have been described (Jonassen et al., 2008; Tadenev et al., 2011). Foxgl ${ }^{\text {Cre }}$ (Hebert and McConnell, 2000) animals were obtained from Jackson Laboratories and were crossed with Ift $20^{\text {flox/flox }}$ mice to inactivate Ift20 in the developing inner ear. Gmap210 (Follit et al., 2008), Ift25 (Keady et al., 2012), Ift27 (Eguether et al., 2014) and Mkks (a gift from Philip Beales, UCL, London, UK) tissue was harvested at embryonic day 18.5 (E18.5) or postnatal day $0(\mathrm{P} 0)$. The morning after mating was considered E0.5 and up to $24 \mathrm{~h}$ after birth was considered P0.

\section{Tissue dissection and immunostaining}

Temporal bones were isolated from embryonic and postnatal mice and fixed in $2-4 \%$ paraformaldehyde at $4{ }^{\circ} \mathrm{C}$ for $2 \mathrm{~h}$. Following microdissection of the cochlea and utricle, immunohistochemistry was performed as described (May-Simera and Kelley, 2012a). Images were obtained using a Zeiss 510 laser scanning confocal microscope. All images were captured from the basal turn of the cochlea unless otherwise stated. Methods used for quantification and additional antibody information are available in the supplementary material methods.

\section{Scanning electron microscopy (SEM), immunogold labeling and transmission electron microscopy (TEM)}

For SEM, temporal bones were dissected and fixed in EM fixative [2.5\% glutaraldehyde (Sigma-Aldrich), 4\% paraformaldehyde (Electron Microscopy Sciences) and $10 \mathrm{mM} \mathrm{CaCl}_{2}$ in HEPES buffer] for $2 \mathrm{~h}$ at room temperature. Organs of Corti were micro-dissected and prepared for SEM using an S-4800 Hitachi electron microscope as described (MaySimera and Kelley, 2012a). For post-embedding immunogold, cochleae from $\mathrm{P} 0$ mice were prepared as described for light microscopy and SEM, and tissue was fixed in 4\% paraformaldehyde and $0.5 \%$ glutaraldehyde in $0.1 \mathrm{M}$ phosphate buffer, and further processed as described (Petralia et al., 2010; Petralia and Wenthold, 1999). Additional details are available in the supplementary material methods. 


\section{Mammalian cell culture and co-IP assays}

Endogenous co-IP of Bbs8 and Ift20 protein was performed using P0 brain lysate harvested in RIPA buffer (Tris- $\mathrm{HCl}, 50 \mathrm{mM} ; \mathrm{NaCl}, 150 \mathrm{mM} ; 1 \% \mathrm{NP}-$ $40,0.5 \%$ sodium deoxycholate, $0.1 \% \mathrm{SDS}, \mathrm{pH} 7.6$ ) containing protease and phosphatase inhibitors (Roche). Anti-Bbs8 and anti-Vangl2 antibodies were used for pull-down or western blot, respectively. For in vitro co-IPs, HEK293 cells were transiently co-transfected with Vangl2 GFP-pCLIg and IFT20Flag plasmids using lipofectamine (Invitrogen). Cell lysates were harvested $48 \mathrm{~h}$ post transfection. Rabbit polyclonal anti-EGFP (Clontech) was used for pull-down, and mouse monoclonal anti-flag was used for western blot. Protein G dynabeads (Invitrogen) were used for co-IP, following the manufacturer's instructions. Post precipitation, protein was harvested in SDS-containing sample loading buffer and detected by western blot. Proteins were run on $4-12 \%$ SDS-PAGE (Invitrogen) using conventional protocols and Pico substrate (Cell Signaling) for chemiluminescent detection.

\section{Affymetrix microarray}

RNA was extracted from two $B b s 8^{-/-}$and two littermate control cochleae at P0 using the RNAqueous-Microkit (Ambion). Two collections were performed on separate days to produce two biological replicates. Total RNA was further purified on an RNAeasy column (Qiagen) and the RNA quality was assessed by an Agilent Bioanalyzer (Agilent Technologies). Target labeling and hybridization to GeneChips were carried out in the NIDDK Microarray Core facility using the GeneChip Mouse 430_2 Array purchased from Affymetrix. The analysis is described in detail in the supplementary materials and methods. The Affymetrix array data have been deposited at ArrayExpress under the accession number E-MTAB-3165.

\section{Acknowledgements}

We would like to acknowledge the editorial assistance of the NIH Fellows Editorial Board for carefully editing the manuscript. We are grateful to Tracy Fitzgerald, Tiansen Li, Chandrakala Puligilla, Kapil Bharti, Tiziana Cogliati, Julien Debbache and Christopher Brinson for technical assistance, scientific reading and commenting on the manuscript.

\section{Competing interests}

The authors declare no competing or financial interests.

\section{Author contributions}

H.L.M.-S. and M.W.K. conceived and designed the experiments; H.L.M.-S., R.S.P., M.M., Y.-X.W. and K.B.S. performed the experiments; and H.L.M.-S. analyzed the data. M.W.K., G.J.P., M.M., R.S.P., M.R.D., Y.L. and W.L. contributed reagents/ materials/analysis tools; and H.L.M.-S., M.W.K. and M.R.D. wrote the paper.

\section{Funding}

This work was supported by intramural and extramural funds from the National Institutes of Health. Intramural funds from the National Institute of Deafness and other Communication Disorders to H.L.M.-S., R.S.P., Y.-X.W., K.B.S. and M.W.K. Intramural funds from the National Eye Institute to H.L.M.-S. Extramural funds from the National Institutes of General Medical Sciences [GM060992 to G.J.P.], National Institute of Neurological Disorders and Stroke [5R01NS055028 to Y.L. and W.L.] National Institute of Deafness and other Communication Disorders [R01DC013066 to M.R.D.] and National Eye Institute [R01EY021146 to M.R.D.]. Intramural INSERM and ANR-14-CE13-0013-01 (M.M.). Deposited in PMC for release after 12 months.

\section{Supplementary material}

Supplementary material available online at

http://dev.biologists.org/lookup/suppl/doi:10.1242/dev.113696//DC1

\section{References}

Ansley, S. J., Badano, J. L., Blacque, O. E., Hill, J., Hoskins, B. E., Leitch, C. C., Kim, J. C., Ross, A. J., Eichers, E. R., Teslovich, T. M. et al. (2003). Basal body dysfunction is a likely cause of pleiotropic Bardet-Biedl syndrome. Nature $\mathbf{4 2 5}$, 628-633.

Aw, S. and Levin, M. (2009). Is left-right asymmetry a form of planar cell polarity? Development 136, 355-366.

Blacque, O. E., Reardon, M. J., Li, C., McCarthy, J., Mahjoub, M. R., Ansley, S. J., Badano, J. L., Mah, A. K., Beales, P. L., Davidson, W. S. et al. (2004) Loss of C. elegans BBS-7 and BBS-8 protein function results in cilia defects and compromised intraflagellar transport. Genes Dev. 18, 1630-1642.
Borovina, A. and Ciruna, B. (2013). IFT88 plays a cilia- and PCP-independent role in controlling oriented cell divisions during vertebrate embryonic development Cell Rep. 5, 37-43.

Carreira-Barbosa, F., Kajita, M., Morel, V., Wada, H., Okamoto, H., Martinez Arias, A., Fujita, Y., Wilson, S. W. and Tada, M. (2009). Flamingo regulates epiboly and convergence/extension movements through cell cohesive and signalling functions during zebrafish gastrulation. Development 136, 383-392.

Copley, C. O., Duncan, J. S., Liu, C., Cheng, H. and Deans, M. R. (2013) Postnatal refinement of auditory hair cell planar polarity deficits occurs in the absence of Vangl2. J. Neurosci. 33, 14001-14016.

Cotanche, D. A. and Corwin, J. T. (1991). Stereociliary bundles reorient during hair cell development and regeneration in the chick cochlea. Hear. Res. 52, 379-402.

Cui, C., Chatterjee, B., Lozito, T. P., Zhang, Z., Francis, R. J., Yagi, H., Swanhart, L. M., Sanker, S., Francis, D., Yu, Q. et al. (2013). Wdpcp, a PCP protein required for ciliogenesis, regulates directional cell migration and cell polarity by direct modulation of the actin cytoskeleton. PLoS Biol. 11, e1001720.

Delaval, B., Bright, A., Lawson, N. D. and Doxsey, S. (2011). The cilia protein IFT88 is required for spindle orientation in mitosis. Nat. Cell Biol. 13, 461-468.

Denman-Johnson, K. and Forge, A. (1999). Establishment of hair bundle polarity and orientation in the developing vestibular system of the mouse. J. Neurocytol. 28, 821-835.

Dickinson, M. E. and McMahon, A. P. (1992). The role of Wnt genes in vertebrate development. Curr. Opin. Genet. Dev. 2, 562-566.

Eguether, T., San Agustin, J. T., Keady, B. T., Jonassen, J. A., Liang, Y., Francis, R., Tobita, K., Johnson, C. A., Abdelhamed, Z. A., Lo, C. W. and Pazour, G. J. (2014). IFT27 links the BBSome to IFT for maintenance of the ciliary signaling compartment. Dev. Cell 31, 279-290.

Ezan, J. and Montcouquiol, M. (2013). Revisiting planar cell polarity in the inner ear. Semin. Cell Dev. Biol. 24, 499-506.

Ezan, J., Lasvaux, L., Gezer, A., Novakovic, A., May-Simera, H., Belotti, E., Lhoumeau, A.-C., Birnbaumer, L., Beer-Hammer, S., Borg, J.-P. et al. (2013) Primary cilium migration depends on G-protein signalling control of subapical cytoskeleton. Nat. Cell Biol. 15, 1107-1115.

Finetti, F., Paccani, S. R., Riparbelli, M. G., Giacomello, E., Perinetti, G., Pazour, G. J., Rosenbaum, J. L. and Baldari, C. T. (2009). Intraflagellar transport is required for polarized recycling of the TCR/CD3 complex to the immune synapse. Nat. Cell Biol. 11, 1332-1339.

Fisch, C. and Dupuis-Williams, P. (2011). Ultrastructure of cilia and flagella - back to the future! Biol. Cell 103, 249-270.

Follit, J. A., Tuft, R. A., Fogarty, K. E. and Pazour, G. J. (2006). The intraflagellar transport protein IFT20 is associated with the Golgi complex and is required for cilia assembly. Mol. Biol. Cell 17, 3781-3792.

Follit, J. A., San Agustin, J. T., Xu, F., Jonassen, J. A., Samtani, R., Lo, C. W. and Pazour, G. J. (2008). The Golgin GMAP210/TRIP11 anchors IFT20 to the Golgi complex. PLoS Genet. 4, e1000315.

Follit, J. A., Xu, F., Keady, B. T. and Pazour, G. J. (2009). Characterization of mouse IFT complex B. Cell Motil. Cytoskeleton 66, 457-468.

Forsythe, E. and Beales, P. L. (2013). Bardet-Biedl syndrome. Eur. J. Hum. Genet. 21, 8-13.

Hébert, J. M. and McConnell, S. K. (2000). Targeting of cre to the Foxg1 (BF-1) locus mediates loxP recombination in the telencephalon and other developing head structures. Dev. Biol. 222, 296-306.

Hernandez-Hernandez, V., Pravincumar, P., Diaz-Font, A., May-Simera, H. Jenkins, D., Knight, M. and Beales, P. L. (2013). Bardet-Biedl syndrome proteins control the cilia length through regulation of actin polymerization. Hum. Mol. Genet. 22, 3858-3868.

Huang, P. and Schier, A. F. (2009). Dampened Hedgehog signaling but normal Wnt signaling in zebrafish without cilia. Development 136, 3089-3098.

Jagger, D., Collin, G., Kelly, J., Towers, E., Nevill, G., Longo-Guess, C., Benson, J., Halsey, K., Dolan, D., Marshall, J. et al. (2011). Alstrom Syndrome protein ALMS1 localizes to basal bodies of cochlear hair cells and regulates ciliumdependent planar cell polarity. Hum. Mol. Genet. 20, 466-481.

Jonassen, J. A., San Agustin, J., Follit, J. A. and Pazour, G. J. (2008). Deletion of IFT20 in the mouse kidney causes misorientation of the mitotic spindle and cystic kidney disease. J. Cell Biol. 183, 377-384.

Jones, C., Roper, V. C., Foucher, I., Qian, D., Banizs, B., Petit, C., Yoder, B. K and Chen, P. (2008). Ciliary proteins link basal body polarization to planar cell polarity regulation. Nat. Genet. $\mathbf{4 0}, 69-77$.

Keady, B. T., Samtani, R., Tobita, K., Tsuchya, M., San Agustin, J. T., Follit, J. A Jonassen, J. A., Subramanian, R., Lo, C. W. and Pazour, G. J. (2012). IFT25 links the signal-dependent movement of Hedgehog components to intraflagella transport. Dev. Cell 22, 940-951.

Kim, S. and Tsiokas, L. (2011). Cilia and cell cycle re-entry: more than a coincidence. Cell Cycle 10, 2683-2690.

Kobayashi, T. and Dynlacht, B. D. (2011). Regulating the transition from centriole to basal body. J. Cell Biol. 193, 435-444

Lee, J. E. and Gleeson, J. G. (2011). A systems-biology approach to understanding the ciliopathy disorders. Genome Med. 3, 59.

Lienkamp, S., Ganner, A. and Walz, G. (2012). Inversin, Wnt signaling and primary cilia. Differentiation 83, S49-S55. 
Lucker, B. F., Behal, R. H., Qin, H., Siron, L. C., Taggart, W. D., Rosenbaum, J. L. and Cole, D. G. (2005). Characterization of the intraflagellar transport complex B core: direct interaction of the IFT81 and IFT74/72 subunits. J. Biol. Chem. 280 27688-27696.

May-Simera, H. and Kelley, M. W. (2012a). Examining planar cell polarity in the Mammalian cochlea. Methods Mol. Biol. 839, 157-171.

May-Simera, H. L. and Kelley, M. W. (2012b). Cilia, Wnt signaling, and the cytoskeleton. Cilia 1, 7

May-Simera, H. L., Ross, A., Rix, S., Forge, A., Beales, P. L. and Jagger, D. J. (2009). Patterns of expression of Bardet-Biedl syndrome proteins in the mammalian cochlea suggest noncentrosomal functions. J. Comp. Neurol. 514 174-188.

May-Simera, H. L., Kai, M., Hernandez, V., Osborn, D. P. S., Tada, M. and Beales, P. L. (2010). Bbs8, together with the planar cell polarity protein Vangl2, is required to establish left-right asymmetry in zebrafish. Dev. Biol. 345, 215-225.

Montcouquiol, M., Rachel, R. A., Lanford, P. J., Copeland, N. G., Jenkins, N. A and Kelley, M. W. (2003). Identification of Vangl2 and Scrb1 as planar polarity genes in mammals. Nature 423, 173-177.

Morsli, H., Choo, D., Ryan, A., Johnson, R. and Wu, D. K. (1998). Development of the mouse inner ear and origin of its sensory organs. J. Neurosci. 18, 3327-3335.

Moser, J. J., Fritzler, M. J., Ou, Y. and Rattner, J. B. (2010). The PCM-basal body/ primary cilium coalition. Semin. Cell Dev. Biol. 21, 148-155.

Nachury, M. V., Loktev, A. V., Zhang, Q., Westlake, C. J., Peränen, J., Merdes, A. Slusarski, D. C., Scheller, R. H., Bazan, J. F., Sheffield, V. C. et al. (2007). A core complex of BBS proteins cooperates with the GTPase Rab8 to promote ciliary membrane biogenesis. Cell 129, 1201-1213.

Narimatsu, M., Bose, R., Pye, M., Zhang, L., Miller, B., Ching, P., Sakuma, R. Luga, V., Roncari, L., Attisano, L. et al. (2009). Regulation of planar cell polarity by Smurf ubiquitin ligases. Cell 137, 295-307.

Ocbina, P. J. R., Tuson, M. and Anderson, K. V. (2009). Primary cilia are not required for normal canonical Wnt signaling in the mouse embryo. PLOS ONE 4 e6839.

Pedersen, L. B., Veland, I. R., Schrøder, J. M. and Christensen, S. T. (2008). Assembly of primary cilia. Dev. Dyn. 237, 1993-2006.

Petralia, R. S. and Wenthold, R. J. (1999). Immunocytochemistry of NMDA receptors. Methods Mol. Biol. 128, 73-92.

Petralia, R. S., Wang, Y. X., Hua, F., Yi, Z., Zhou, A., Ge, L., Stephenson, F. A. and Wenthold, R. J. (2010). Organization of NMDA receptors at extrasynaptic locations. Neuroscience 167, 68-87.

Robert, A., Margall-Ducos, G., Guidotti, J.-E., Bregerie, O., Celati, C., Brechot, C. and Desdouets, C. (2007). The intraflagellar transport component IFT88 polaris is a centrosomal protein regulating G1-S transition in non-ciliated cells. J. Cell Sci. 120, 628-637.

Ross, A. J., May-Simera, H., Eichers, E. R., Kai, M., Hill, J., Jagger, D. J., Leitch, C. C., Chapple, J. P., Munro, P. M., Fisher, S. et al. (2005). Disruption of BardetBiedl syndrome ciliary proteins perturbs planar cell polarity in vertebrates. Nat. Genet. 37, 1135-1140.
Saburi, S. and McNeill, H. (2005). Organising cells into tissues: new roles for cell adhesion molecules in planar cell polarity. Curr. Opin. Cell Biol. 17, 482-488.

Sedmak, T. and Wolfrum, U. (2010). Intraflagellar transport molecules in ciliary and nonciliary cells of the retina. J. Cell Biol. 189, 171-186.

Simons, M. and Mlodzik, M. (2008). Planar cell polarity signaling: from fly development to human disease. Annu. Rev. Genet. 42, 517-540.

Simons, M., Gloy, J., Ganner, A., Bullerkotte, A., Bashkurov, M., Krönig, C., Schermer, B., Benzing, T., Cabello, O. A., Jenny, A. et al. (2005). Inversin, the gene product mutated in nephronophthisis type II, functions as a molecular switch between Wnt signaling pathways. Nat. Genet. 37, 537-543.

Sipe, C. W. and Lu, X. (2011). Kif3a regulates planar polarization of auditory hair cells through both ciliary and non-ciliary mechanisms. Development 138 3441-3449.

Tadenev, A. L. D., Kulaga, H. M., May-Simera, H. L., Kelley, M. W., Katsanis, N. and Reed, R. R. (2011). Loss of Bardet-Biedl syndrome protein-8 (BBS8) perturbs olfactory function, protein localization, and axon targeting. Proc. Natl. Acad. Sci. USA 108, 10320-10325.

Tissir, F., Qu, Y., Montcouquiol, M., Zhou, L., Komatsu, K., Shi, D., Fujimori, T., Labeau, J., Tyteca, D., Courtoy, P. et al. (2010). Lack of cadherins Celsr2 and Celsr3 impairs ependymal ciliogenesis, leading to fatal hydrocephalus. Nat. Neurosci. 13, 700-707.

Tobin, J. L., Di Franco, M., Eichers, E., May-Simera, H., Garcia, M., Yan, J. Quinlan, R., Justice, M. J., Hennekam, R. C., Briscoe, J. et al. (2008). Inhibition of neural crest migration underlies craniofacial dysmorphology and Hirschsprung's disease in Bardet-Biedl syndrome. Proc. Natl. Acad. Sci. USA 105, 6714-6719.

van Dam, T. J. P., Wheway, G., Slaats, G. G.SYSCILIA Study Group, Huynen, M. A. and Giles, R. H. (2013). The SYSCILIA gold standard (SCGSv1) of known ciliary components and its applications within a systems biology consortium. Cilia 2,7 .

Veland, I. R., Montjean, R., Eley, L., Pedersen, L. B., Schwab, A., Goodship, J. Kristiansen, K., Pedersen, S. F., Saunier, S. and Christensen, S. T. (2013). Inversin/Nephrocystin-2 is required for fibroblast polarity and directional cell migration. PLOS ONE 8, e60193.

Vladar, E. K., Antic, D. and Axelrod, J. D. (2009). Planar cell polarity signaling: the developing cell's compass. Cold Spring Harb. Perspect. Biol. 1, a002964.

Wallingford, J. B. and Mitchell, B. (2011). Strange as it may seem: the many links between Wnt signaling, planar cell polarity, and cilia. Genes Dev. 25, 201-213.

Wang, Y., Guo, N. and Nathans, J. (2006). The role of Frizzled3 and Frizzled6 in neural tube closure and in the planar polarity of inner-ear sensory hair cells. J. Neurosci. 26, 2147-2156.

Waters, A. M. and Beales, P. L. (2011). Ciliopathies: an expanding disease spectrum. Pediatr. Nephrol. 26, 1039-1056.

Yuan, S. and Sun, Z. (2013). Expanding horizons: ciliary proteins reach beyond cilia. Annu. Rev. Genet. 47, 353-376. 